\title{
Evaluation of Pooled Sample Analysis Strategy for SARS-CoV-2 RT-PCR Tests in Diagnosis and Screening of COVID-19
}

\author{
S GITI $^{\mathrm{a}}$, M HOQUE $^{\mathrm{b}}$, AA KHAN $^{\mathrm{c}}$, MMH SHOUROV $^{\mathrm{d}}$
}

\begin{abstract}
:
Introduction: The recent outbreak of COVID-19 is a serious global concern. The emergence of SARS-CoV-2 led to a current pandemic of unprecedented levels. Early detection of the infection and prompt isolation of the cases are fundamental for containment of such outbreak. By this time, healthcare systems are facing shortages of reagents for diagnosing this disease. In this context, sample pooling can be an effective strategy to overcome this situation. This study aimed to evaluate the effectiveness of sample pooling strategy for RT-PCR tests for diagnosing and screening of COVID19 in mass population.
\end{abstract}

Methods: A cross-sectional study was performed at the COVID-19 laboratory of Armed Forces Institute of Pathology of Bangladesh to evaluate the efficacy of sample pooling technique for detecting SARS-CoV-2 infection. In this laboratory, nasopharyngeal and oropharyngeal swabs were taken for the RT-PCR test to diagnose COVID-19. For each patient, both nasopharyngeal and oropharyngeal samples were taken and then mixed to make a single sample. Pooling

Introduction:

Coronavirus disease 2019 (COVID-19) is an infectious disease caused by severe acute respiratory syndrome

a. Maj Gen Susane Giti, MBBS, MCPS (Clinical Pathology), FCPS (Haematology), MMEd. Commandant, Armed Forces Institute of Pathology, Dhaka Cantonment.

b. Col Md Monirul Hoque, MBBS, DCP, MCPS (Clinical Pathology), FCPS (Microbiology). Classified Specialist in Pathology, Armed Forces Institute of Pathology, Dhaka Cantonment.

c. Brig Gen Arif Ahmed Khan, MBBS, MCPS (Clinical Pathology), FCPS (Microbiology). Adviser Specialist in Pathology and Deputy Commandant, Armed Forces Institute of Pathology, Dhaka Cantonment.

d. Maj Md Mehedhi Hasan Shourov, MBBS, DCP, MCPS (Clinical Pathology). Graded Specialist in Pathology, Armed Forces Institute of Pathology, Dhaka Cantonment.

Address of Correspondence: Col Md Monirul Hoque, MBBS, DCP, MCPS (Clinical Pathology), FCPS (Microbiology). Classified Specialist in Pathology, Armed Forces Institute of Pathology, Dhaka Cantonment. E-mail: mmhoque21@yahoo.com. was performed from the samples collected from 1 April 2020 to 30 April 2020. Total 350 samples were distributed randomly in 70 pools, so that each pool contains 5 samples. Positive pools were deconvulated and each sample was tested separately. Screening was performed by using RT-PCR targeting the ORF-1ab Region and $\mathrm{N}$-gene.

Results: Out of 70 pools 16 (22.85\%) were found positive. Eighty samples of these 16 pools were tested individually and $21(21 / 80,26.25 \%)$ samples were found positive. All the positive pools were reproducible with testing of the individual samples of that pool (100\%).

Conclusion: Strategies of using pooled samples for screening may facilitate detection of early community transmission of SARS-CoV-2 and enable timely implementation of appropriate infection control measures to reduce spread.

Keywords: SARS-CoV-2, COVID-19, RT-qPCR.

(J Bangladesh Coll Phys Surg 2020; 38: 16-20) DOI: https://doi.org/10.3329/jbcps.v38i0.47349

coronavirus 2 (SARS-CoV-2). ${ }^{1}$ It was first identified in December 2019 in Wuhan, China. Already it has spread globally and caused the ongoing pandemic. ${ }^{2}$

As of 10 May 2020, more than 4.02 million cases have been reported across 187 countries and territories, resulting in more than 279,000 deaths. By this time more than 1.37 million people have recovered. ${ }^{3}$

Common symptoms include fever, cough, fatigue, shortness of breath, and loss of smell and taste. About $80 \%$ patients recover from the disease without needing hospital treatment. Around 1 out of every 5 people who gets COVID-19 becomes seriously ill and develops difficulty in breathing, acute respiratory distress syndrome (ARDS), multi-organ failure, septic shock, and blood clots. The incubation period is typically around five days but may range from two to fourteen days. ${ }^{4}$

The virus is primarily spread between people during close contact, most often via small droplets produced 
by coughing, sneezing, and talking. The droplets usually fall to the ground or onto surfaces rather than travelling through air over long distances. Less commonly, people may also become infected by touching a contaminated surface and then touching their face. ${ }^{5}$ The standard method of diagnosis is by real-time reverse transcription polymerase chain reaction (rRT-PCR) from a nasopharyngeal swab or oropharyngeal swab. ${ }^{6}$

Recently almost every nation of the world is facing the challenge of the COVID-19 outbreak. Healthcare systems all over the world are experiencing much difficulties to contain this unprecedented pandemic of SARS-CoV-2 pandemic. ${ }^{7}$

The fundamental response measure to combat this pandemic is early detection of the case which enables the quarantine of COVID-19 patients to prevent local spread, and more broadly informs national response measures. ${ }^{8}$

Because of limited monitoring capacity, testing in most countries is generally focused on acutely ill patients, while potentially infectious carriers at the community remain undiagnosed. As many countries are already experiencing shortages of diagnosis kits and factories struggling to keep with the demand, it has become important to come up with new ways to conserve the reagents used for diagnostic tests. At the same time, as the disease is novel, it is of value to validate any modifications to the testing process before universal adoption 9

Pooling method was first suggested by Dorfman in 1943. It was then perfected over the years. In this method samples are mixed and tested at a single pool, and subsequent individual tests are made only if the pool tests positive. ${ }^{10,11}$

We frequently apply pooling diagnostic technique in other infectious diseases and it is especially advantageous as it requires no additional training, equipment, or materials.

In addition to being used in the clinic for infectious disease diagnostics in previous epidemics , pooling has been proven to work for RT-qPCR, a timeconsuming step for which the reagents are expected to be in short supply.$^{12}$

SARS-CoV-2 is a novel pathogen and it is still unclear how diluting a sample containing its RNA would affect the sensitivity of this assay and the false-negative rate.
In this study we applied sample pooling strategy in our COVID-19 laboratory for diagnosing and screening of SARS-CoV-2 infection.

\section{Materials \& methods}

Method of study

It is a cross-sectional study.

Study period

01 April 2020 to 30 April 2020.

\section{Sample collection}

For each patient both nasopharyngeal and oropharyngeal swabs were collected with the synthetic fibre swab sticks. After collection the swab sticks were kept in the liquid viral transport media and then sent to the COVID-19 laboratory of AFIP, Dhaka Cantonment.

A volume of 10 microliter of the sample was mixed with 10 microliter lysis buffer and this mixture was kept 15 minutes for getting extracted RNA.

\section{Individual RT-PCR tests in the clinical laboratory}

RT-PCR was performed in our laboratory to detect the presence of SARS-CoV-2 RNA with Sansure Biotech nCoV Nucleic Acid Detection Kit in Rotor Gene-Q Qiagen qPCR Machine. Reactions were heated to $50^{\circ} \mathrm{C}$ for 30 minutes for reverse transcription, denatured at $95^{\circ} \mathrm{C}$ for 10 minutes and then 45 cycles of amplification were carried in $95^{\circ} \mathrm{C}$ for 15 seconds and $55^{\circ} \mathrm{C}$ for 32 seconds. Fluorescence was measured using the FAM, ROX and Cy5 parameters.

\section{Pooled-samples RT-PCR in the research laboratory} Laboratory RT-PCR procedure was performed according to the procedure for individual samples in the clinical laboratory, on an identical qPCR machine and program and with fresh reagents.

\section{Pooling}

To conserve reagents we pooled the samples randomly into total 70 groups where each group contains 05 samples.

\section{Ethical approval}

This study was granted by the ethical approval committee.

\section{Results:}

A total of 350 samples were screened by making 70 pools each of which containing 05 samples. Among these 70 
pools 16 were found positive. The positivity rate was $22.85 \%$ (Chart-1). The highest number of pooled sample were tested in $3^{\text {rd }}$ week of the month (Chart-2). These 16 positive pools were then tested as per principle of pooling strategy. Eighty samples of these 16 pools were tested individually in the same RT-PCR machine with similar technique and 21 samples were found positive (Chart-3). The positivity rate among these 80 samples was $26.25 \%$. In total, out of 350 samples $6.0 \%$ was positive (Chart-4). The most number of positive samples (15) were in $4^{\text {th }}$ week of the month. All the positive pools were reproducible with testing of the individual samples of that pool $(100 \%)$.

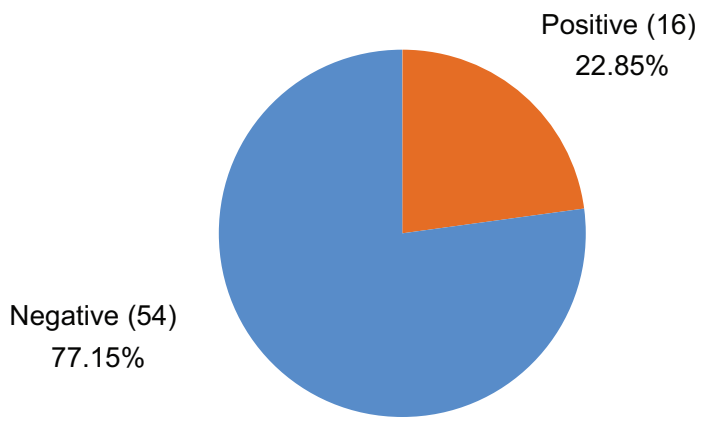

Fig.-1: The rate of positivity among all pools $(n=70)$.

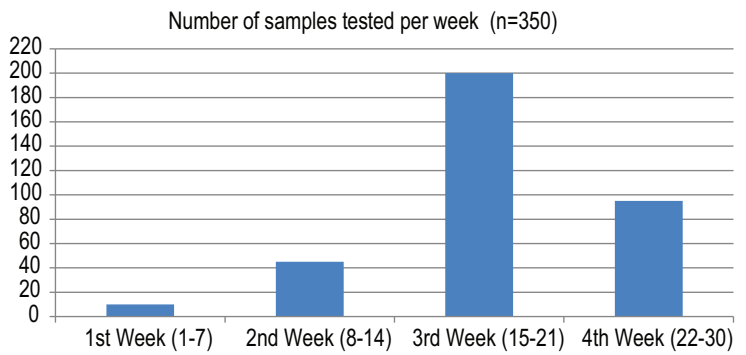

Fig.-2: Number samples tested weekly $(n=350)$.

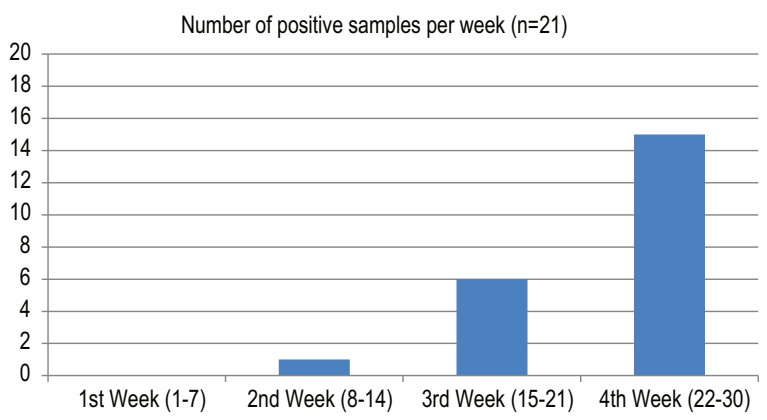

Fig.-3: Distribution of positive cases per week $(n=21)$.
Rate of positivity among all samples $(n=350)$

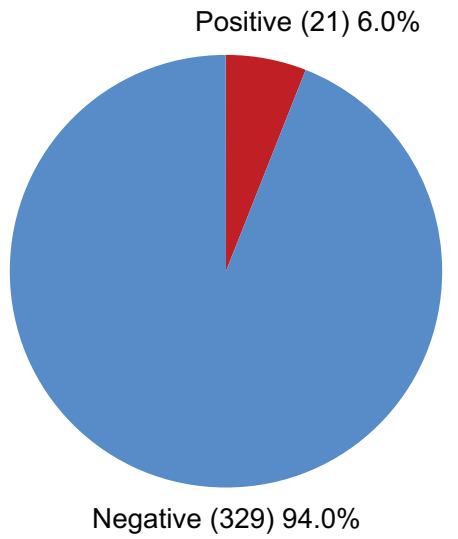

Fig.-4: The rate of positivity among all samples $(n=350)$.

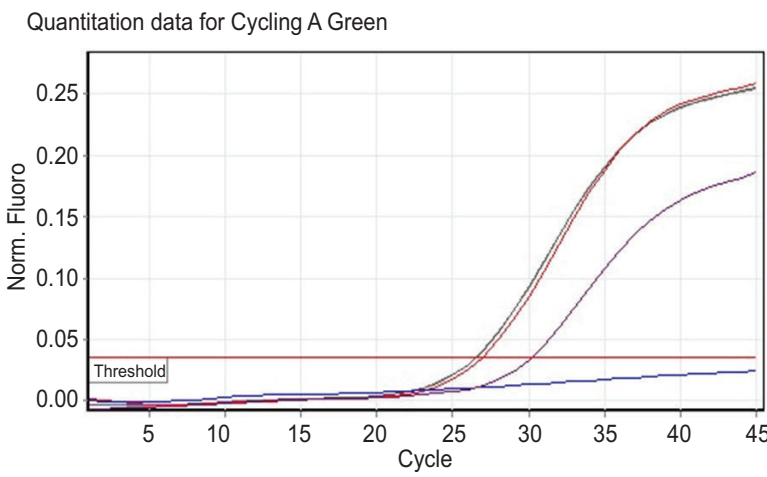

Fig.-5: Amplification curve of 03 pools along with positive control and negative control (PS-1, PS-2, PS3, PC and NC). All three pools and PC showed characteristic sigmoid amplification curve. Channel : Green (FAM); Target : ORF-1ab Region.

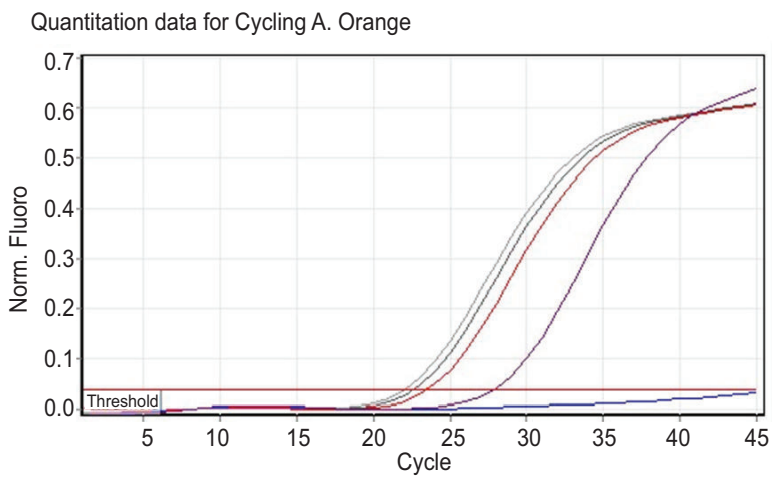

Fig.-6: Amplification curve of 03 pools along with positive control and negative control (PS-1, PS-2, PS3, PC and NC). All three pools and PC showed characteristic sigmoid amplification curve. Channel : Orange (ROX); Target : N-Gene. 


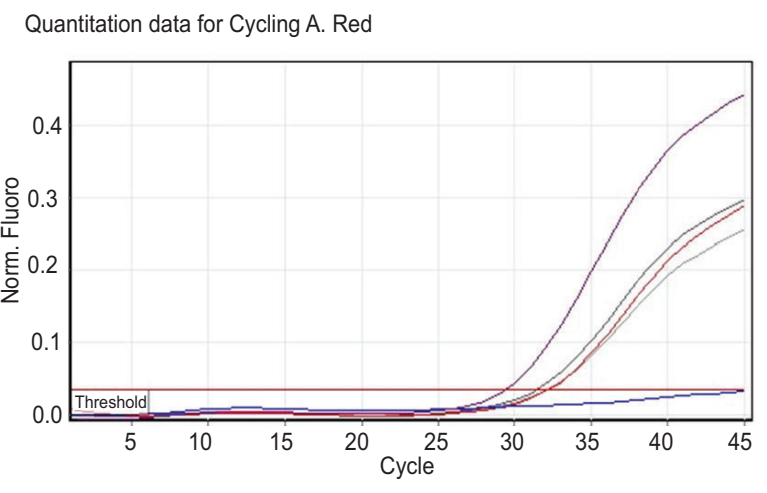

Fig.-7: Amplification curve of 03 pools along with positive control and negative control (PS-1, PS-2, PS3, PC and NC). All three pools and PC showed characteristic sigmoid amplification curve. Channel: Red (Cy5); Target : Internal Control.

\section{Discussion:}

The recently emerged SARS-CoV-2 virus has already turned into a global pandemic. Each day the number of affected persons and the death toll is increasing rapidly. As this virus transmits through person to person by air droplets discharged from infected person, the prime measure to prevent the spreading of this virus is to identify the carrier and contacts as soon as possible and subsequent isolation of those persons. To ensure this adequate and widespread testing facilities is the most important task to be accomplished.

COVID-19 pandemic has revealed the global importance of robust diagnostic testing to differentiate severe acute respiratory syndrome coronavirus 2 (SARS-CoV-2) from other routine respiratory infections and guide appropriate clinical management. ${ }^{13}$

As this coronavirus is a novel virus and it has spread in almost every corner of the globe within a period of time less than 02 months, all healthcare facilities across the world are facing the scarcity of reagents and other equipments for diagnosing this infection. In this context sample pooling strategy can be an excellent way to meet the necessity of mass screening and diagnosis purposes. Moreover, we can minimize the costs of reagents by applying this technique in a resource-limited developing country like Bangladesh. ${ }^{14}$

In Israel, Idan Yelin et al. conducted a study to test the ability of the standard RT-qPCR test for detecting a positive sample within a random pool of negative samples. Pooling clinical RNA samples, they tested previously confirmed positive samples alone and combined with an increasing number of previously confirmed negative samples. They found that positive samples can still be well observed in pools of up to 32 samples, and possibly even 64 with additional PCR cycles. ${ }^{14}$

In USA, Catherine A. Hogan et al. conducted a retrospective study regarding the evaluation of efficacy of pooling strategy for SARS-CoV-2 RT-PCR with a good number of samples. They screened a total of 292 pools, corresponding with 2740 nasopharyngeal samples and 148 bronchoalveolar lavage samples. The confirmed positivity rate for SARS-CoV- 2 was $0.07 \%(2 / 2888)$. The 2 positive samples showed detection of $\mathrm{E}$ and $\mathrm{RdRp}$. Only 1 pool showed a positive E signal that was not reproducible with testing of the individual samples of that pool. ${ }^{15}$ In comparison of this study, we found 16 pools positive among 70 corresponding to 350 samples $(22.85 \%)$. After individual testing of the samples of these 16 positive pools, 21 samples were positive $(26.25 \%)$. Among total 350 samples $6.0 \%$ was positive. All the positive pools were reproducible with testing of the individual samples of that pool (100\%).

The findings of our study perfectly match with the aforementioned two studies in context of evaluating the applicability and efficacy of sample pooling technique to conserve the reagents and manpower for COVID-19 RT-PCR tests.

A pooled screening strategy was pursued to increase testing throughput, limit use of reagents, and minimize the expenditure of the tests in a resource-limited laboratory facility.

Due to the challenges of restricted access to diagnostic tests and kit supplies across the globe, early testing has largely been limited to symptomatic individuals fulfilling testing criteria. ${ }^{16}$

Although this approach facilitates rational use of resources, this study is limited in that it was performed in a single laboratory in a restricted geographical area. Additional data are thus required to validate this approach on a larger scale.

\section{Conclusion:}

Pooled-sample RT-PCR analysis strategies will save substantial resources and manpower for COVID-19 mass 
testing. In particular, it can facilitate mass screening in early stages of COVID-19 outbreaks. In practical, determining carriage frequency in the population is the mainstay of local and global epidemic response which is greatly enabled by pooling techniques. Furthermore, pooling techniques can be used for routine monitoring of essential work groups, such as hospital staff, military units, and factory workers. Moreover, this screening strategy does not obviate the need for individual diagnostic testing, particularly as community transmission intensifies.

Conflict of Interest: We have no conflict of interest.

\section{References:}

1. "COVID-19 Dashboard by the Center for Systems Science and Engineering (CSSE) at Johns Hopkins University (JHU)". ArcGIS. Johns Hopkins University. Retrieved 10 May 2020 .

2. "Coronavirus disease 2019 (COVID-19)—Symptoms and causes”. Mayo Clinic. Retrieved 14 April 2020.

3. Nussbaumer-Streit B, Mayr V, Dobrescu AI, Chapman A, Persad E, Klerings I, et al. (April 2020). "Quarantine alone or in combination with other public health measures to control COVID-19: a rapid review". The Cochrane Database of Systematic Reviews. doi:10.1002/14651858. CD013574. PMC 7141753. PMID 32267544.

4. https://www.who.int/emergencies/diseases/novelcoronavirus-2019/question-and-answers-hub/q-a-detail/q-acoronaviruses.

5. "Symptoms of Coronavirus". U.S. Centers for Disease Control and Prevention (CDC). 20 March 2020. Archived from the original on 30 January 2020.

6. "Interim Guidelines for Collecting, Handling, and Testing Clinical Specimens from Persons for Coronavirus Disease 2019 (COVID-19)". Centers for Disease Control and Prevention (CDC). 11 February 2020. Archived from the original on 4 March 2020. Retrieved 26 March 2020.

7. Huang G, Zeng W, Wang W, Song Y, Mo X, Li J, et al. Triaging patients in the outbreak of the 2019 novel coronavirus. medRxiv. 2020; 2020.03.13.20035212.
8. CDC. Coronavirus Disease 2019 (COVID-19) Situation Summary. In: Centers for Disease Control and Prevention [Internet]. 16 Mar 2020 [cited 17 Mar 2020]. Available: https://www.cdc.gov/coronavirus/2019-ncov/cases-updates/ summary.html

9. CDC. Research Use Only Real-Time RT-PCR Protocol for Identification of 2019-nCoV. In: Centers for Disease Control and Prevention [Internet]. 9 Mar 2020 [cited 17 Mar 2020]. Available: https://www.cdc.gov/coronavirus/2019-ncov/ about/testing.html

10. Dorfman R. The Detection of Defective Members of Large Populations. Ann Math Stat. 1943;14: 436-440.

11. Bilder CR, Tebbs JM. Pooled-testing procedures for screening high volume clinical specimens in heterogeneous populations. Stat Med. 2012;31: 3261-3268.

12. Herper M, Begley S, Boodman E, Robbins R, Facher L. Thermo Fisher to produce millions of coronavirus diagnostic tests - STAT. In: STAT [Internet]. 14 Mar 2020 [cited 17 Mar 2020]. Available: https://www.statnews.com/ 2020/03/14/thermo-fisher-to-produce-millions-ofcoronavirus-dia gnostic-tests.

13. Sharfstein JM, BeckerSJ, Mello MM. Diagnostic testing for the novel coronavirus. JAMA. Published online March 9, 2020. doi:10.1001/jama.2020.3864.

14. Yelin I, Aharony N,Tamar ES, Argoetti A, Messer E, Berenbaum D, Shafran E, Kuzli A, Gandali N, Hashimshony T, Gutfreund YM, Halberthal M, Geffen Y, Szwarcwort-Cohen M, Kishony R. Evaluation of COVID-19 RT-qPCR test in multi-sample pools. MEDRXIV. https://doi.org/10.1101/2020.03.26.20039438.

15. Hogan CA, Sahoo MK, Pinsky BA. Sample Pooling as a Strategy to Detect Community Transmission of SARSCoV-2. JAMA. Published online April 6, 2020. doi:10.1001/ jama.2020.5445.

16. Deckert A, Barnighausen T, Kyei N. Pooled-sample analysis strategies for COVID-19 mass testing: a simulation study. ResearchGate. Published online: 2 April 2020. DOI: 10.2471/BLT.20.257188. 